\title{
One hundred million years of interhemispheric communication: the history of the corpus callosum
}

F. Aboitiz and J. Montiel

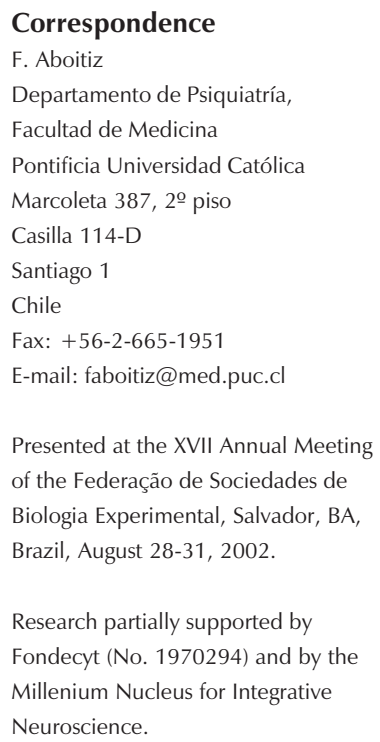

Presented at the XVII Annual Meeting of the Federação de Sociedades de Biologia Experimental, Salvador, BA, Brazil, August 28-31, 2002.

Research partially supported by Fondecyt (No. 1970294) and by the Millenium Nucleus for Integrative Neuroscience.

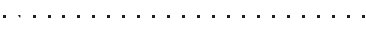

Departamento de Psiquiatría y Centro de Investigaciones Médicas, Facultad de Medicina, Pontificia Universidad Católica de Chile, and Millenium Nucleus for Integrative Neuroscience, Santiago, Chile
Abstract

Analysis of regional corpus callosum fiber composition reveals that callosal regions connecting primary and secondary sensory areas tend to have higher proportions of coarse-diameter, highly myelinated fibers than callosal regions connecting so-called higher-order areas. This suggests that in primary/secondary sensory areas there are strong timing constraints for interhemispheric communication, which may be related to the process of midline fusion of the two sensory hemifields across the hemispheres. We postulate that the evolutionary origin of the corpus callosum in placental mammals is related to the mechanism of midline fusion in the sensory cortices, which only in mammals receive a topographically organized representation of the sensory surfaces. The early corpus callosum may have also served as a substrate for growth of fibers connecting higher-order areas, which possibly participated in the propagation of neuronal ensembles of synchronized activity between the hemispheres. However, as brains became much larger, the increasingly longer interhemispheric distance may have worked as a constraint for efficient callosal transmission. Callosal fiber composition tends to be quite uniform across species with different brain sizes, suggesting that the delay in callosal transmission is longer in bigger brains. There is only a small subset of large-diameter callosal fibers whose size increases with increasing interhemispheric distance. These limitations in interhemispheric connectivity may have favored the development of brain lateralization in some species like humans.

"...if the currently received statements are correct, the appearance of the corpus callosum in the placental mammals is the greatest and most sudden modification exhibited by the brain in the whole series of vertebrated animals..." T.H. Huxley (1).

\section{Introduction}

The corpus callosum is a unique feature of the brain of placental mammals, so much so that we may state that it can be a diagnostic character of placentals just like the very placenta is. This structure was first described
Key words

- Commissures

- Evolution

- Interhemispheric transfer

- Lateralization

- Synchronization 
unique to placentals, since the dorsal commissure originated mainly from the hippocampal formation, while the corpus callosum had no relation to the hippocampus. The absence of any kind of callosal primordia in nonplacental mammals implied a sudden evolutionary origin of the corpus callosum, with no structures ancestral to it. This was unexpected according to Darwin's concept of evolution by the successive accumulation of small changes. In fact, even Huxley, a champion of gradualism, would admit in 1863 that the origin of the corpus callosum signified a major leap in evolution. In 1865 , some thirty years later than Owen, Flower (2) and later Smith (3) argued that both the corpus callosum of placentals and the dorsal commissure of marsupials contained fibers connecting the anterior part of the mesial hemisphere and consequently were partly homologues. This observation would agree with the concept of a gradual origin of the corpus callosum, since fibers that originally traveled through the dorsal commissure might have found a different route across the hemispheres. However, Flower went perhaps too far in naming the dorsal commissure of marsupials as the corpus callosum, and proposing that the corpus callosum could be found even in reptiles and other "lower" vertebrates. Indeed, when saying this, he was actually speaking of the dorsal hippocampal commissure. For this reason, Flower later became strongly discredited (3). Nevertheless, Flower may have been right in that some neocortical fibers might cross through the hippocampal commissure in marsupials, which would explain its large size.

Aside from these historical considerations, the corpus callosum remains an evolutionary puzzle. Mutations producing the absence of the corpus callosum are not uncommon, and if there were no adaptive value in this structure it would be common to find placental lineages in which the corpus callosum had been lost. In addition, it is surprising that few dramatic long-term effects beside the callosal discon- nection syndrome are seen after section of the corpus callosum in humans and in other animals $(4,5)$. Therefore, the question arises about the adaptive value of the largest fiber tract in the placental nervous system. Which circumstances were involved in the origin of the corpus callosum, and why was it maintained in the history of placental mammals? Furthermore, is the corpus callosum related to the emergence of brain lateralization in humans and to conscious experience? (4) In this paper, we will discuss our studies on the comparative fiber composition of the corpus callosum, which in our view may provide important insights into the problems raised above.

\section{Fiber composition of the corpus callosum}

The corpus callosum is not a homogeneous structure in the sense that fibers connecting specific cortical areas travel through distinct callosal regions. In other words, there is a rough topographic representation of the different cortical areas along the corpus callosum, in which anterior cortical areas are connected through the anterior corpus callosum, and more posterior areas are connected through more posterior regions. This topography is more accurate in species like the macaque (6), but in rodents it becomes more diffuse (7). Topographic variability implies that there may be concomitant regional differences in terms of callosal fiber composition, as the kind of information transmitted differs across different cortical areas. In fact, analyses of fiber composition in the primate corpus callosum reveal a wide diversity of fiber calibers, which are distributed in an uneven manner along this structure (see Figure $1 ; 8,9)$. Callosal regions connecting prefrontal and temporoparietal association areas are characterized by large proportions of poorly myelinated, small caliber, slow-conducting fibers, while in regions connecting primary and secondary sensorimotor areas there is a concentration of fast-conducting, 
highly myelinated fibers of more than $3 \mu \mathrm{m}$ in diameter. However, in other mammals such as carnivores, rodents and ungulates, we did not observe regional differences in fiber size, at least in the posterior corpus callosum (10). This may partly reflect the more diffuse topographic arrangement of different cortical areas in the corpus callosum of these species (see Ref. 10,11).

\section{Functions of different callosal fibers}

The uneven distribution of fiber types along the corpus callosum suggests important functional differences in interhemispheric communication between different types of cortical areas. First, it is important to recall that many callosal projections are homotopic, that is, they connect equivalent regions between the two hemispheres (12). This is not to say that there are no heterotopic callosal projections (i.e., connecting different areas across the hemispheres). In the visual cortex, callosal cells and fibers connecting lower-order areas (V1, V2, V3) with a visuotopic organization tend to be concentrated on the borders between these areas (Figure 2). More specifically, many callosal-projecting cells and their terminals are located in a stripe corresponding to the representation of the visual field's midline (Figure 3); these cells can be found both in superficial and deep cortical layers $(12,13)$. Since each hemisphere contains a representation of the contralateral visual hemifield, callosal fibers have been proposed to connect the two hemirepresentations of the whole visual field at the level of the midline. On the border between the primary and secondary visual areas of the cat and other mammals there is a band containing two mirror images (one in V1 and the other in V2) of the central, binocular visual field; each image has an ipsilateral and a contralateral representation of the central visual field (14-16). In this region, many callosal cells connect points of the contralateral visual representa-
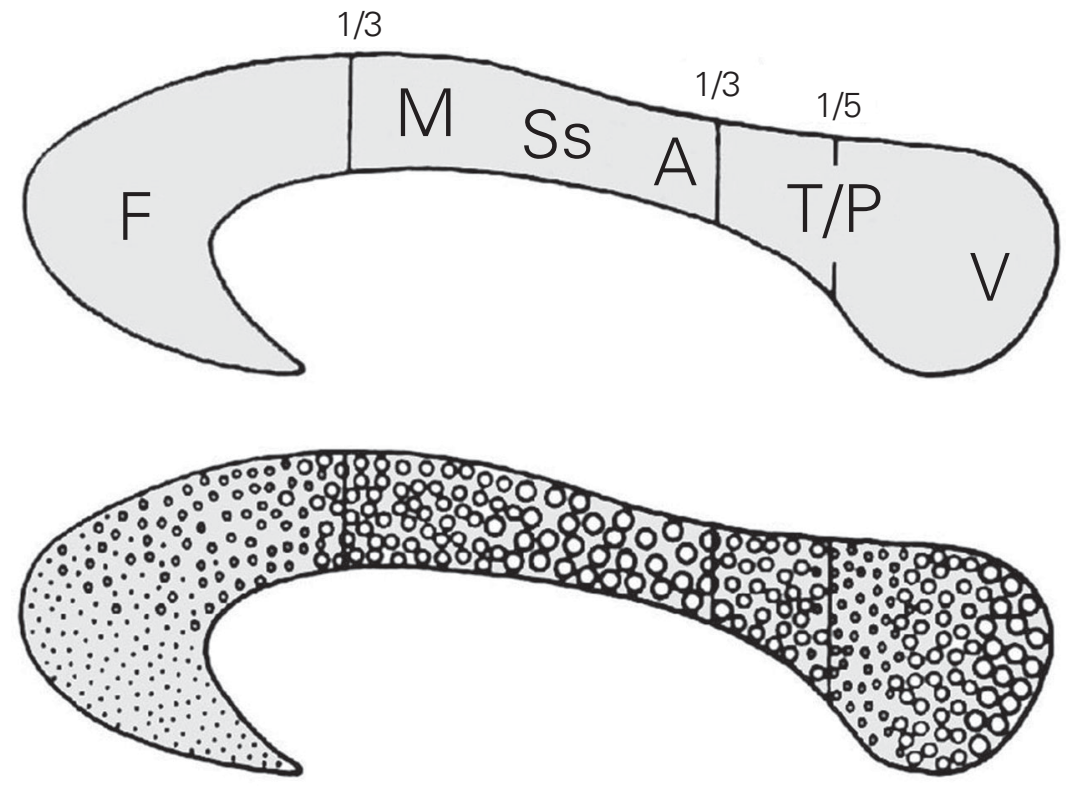

Figure 1. Cross-section of the human corpus callosum indicating the representation of different cortical regions (top). Regional differences in fiber composition along the corpus callosum (larger circles indicate larger fiber diameters) (bottom). A, auditory fibers; F, frontal fibers; $M$, motor cortex fibers; Ss, somatosensory fibers; $T / P$, temporoparietal fibers; $V$, visual fibers.
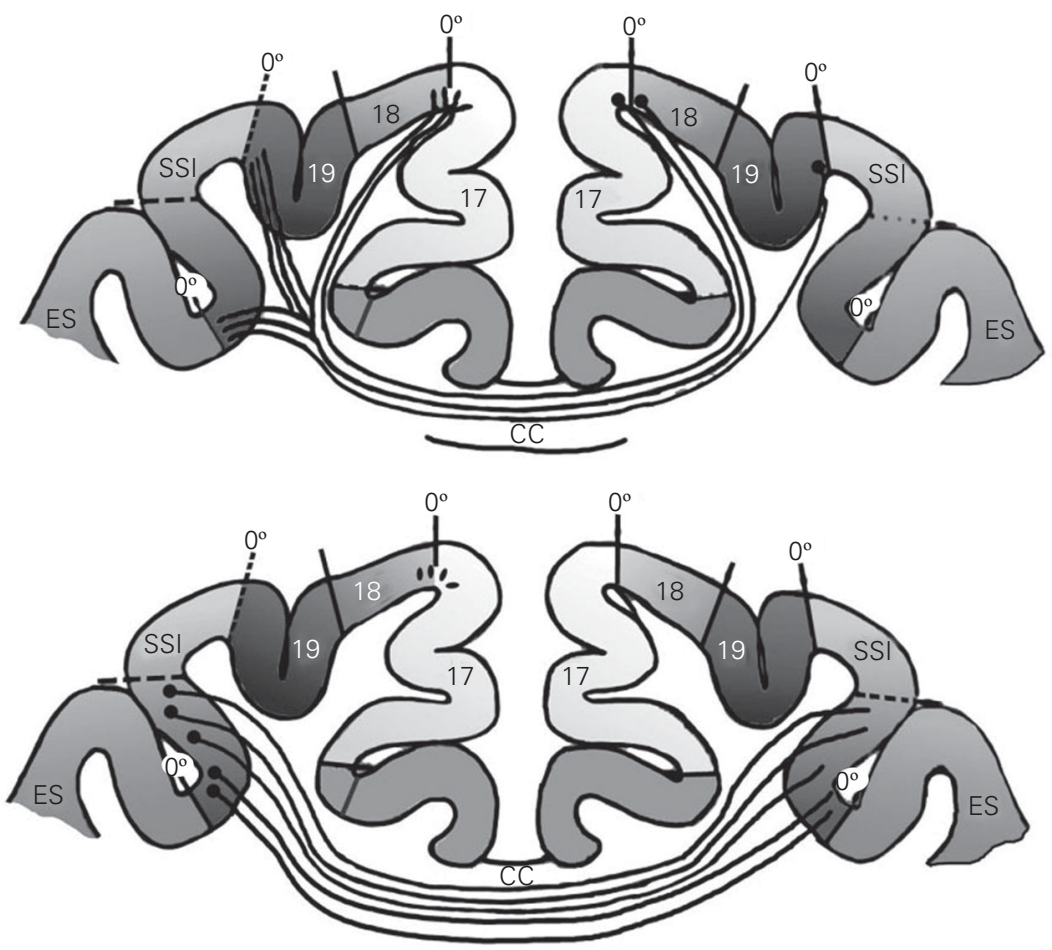

Figure 2. Diagrams indicating the distribution of callosal fibers in different cortical areas of the cat. Top, Fibers originating at the $17 / 18$ border project to several regions representing visually equivalent points along the midline (0 degree). Bottom, Fibers connecting higherorder areas (ectosylvian cortex, ES) originate modified from and project to the whole contralateral area. CC, corpus callosum; SSI, suprasylvian cortex. Modified from Ref. 17. 
tion of one hemisphere with regions representing the same or a neighboring visual position in the ipsilateral representation of the other hemisphere (see Figure 3). In addition, in V1 and V2, a few cells in deep layer $\mathrm{V}$ have been found to project through the corpus callosum from regions well within the respective areas, representing peripheral regions of the visual field (18). The explanation for these cells is not clear yet, but may be related to anticipation of trajectories of fast-

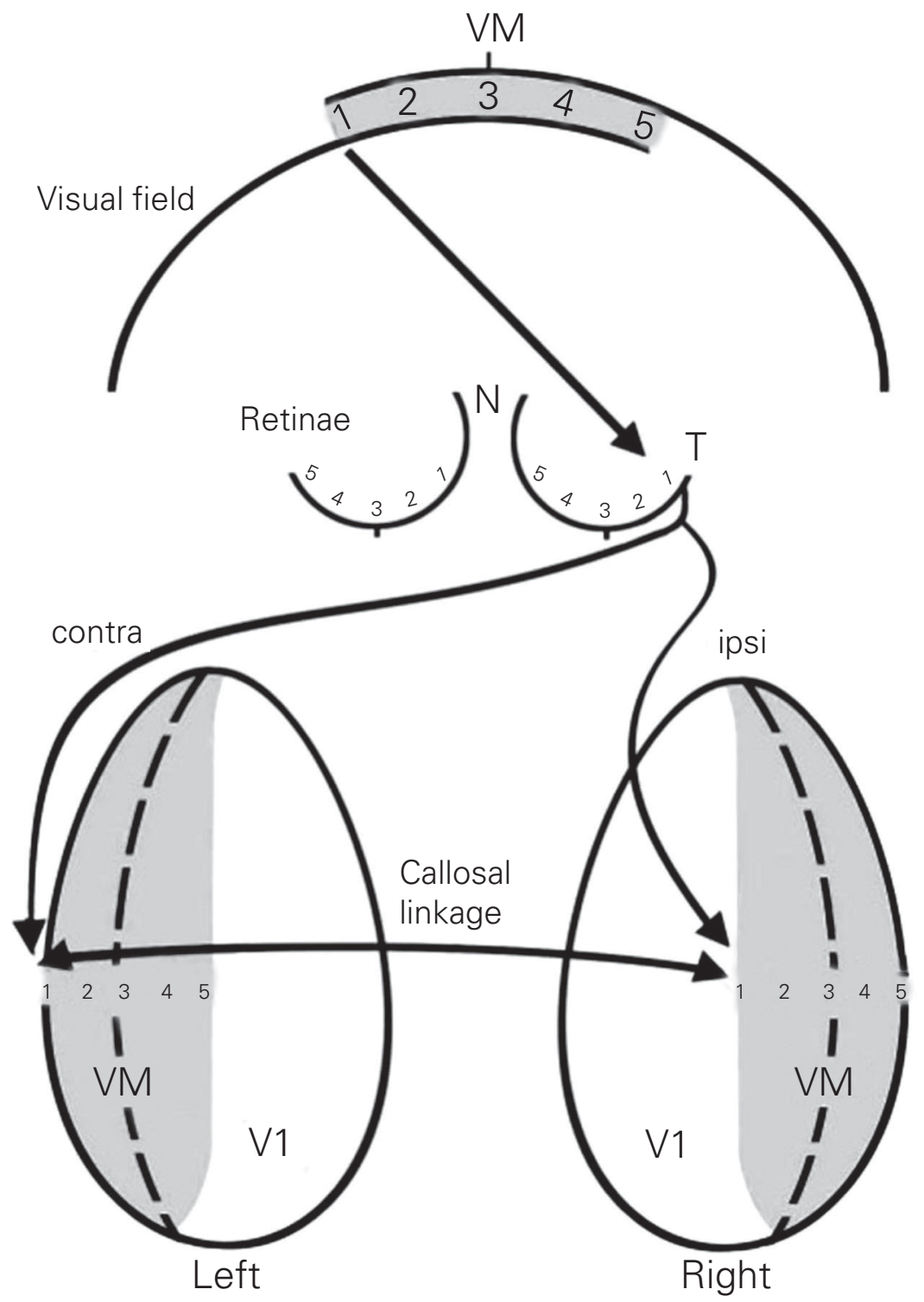

Figure 3. In V1, callosal fibers connect contralateral ocular dominance columns with ipsilateral ocular dominance columns, representing visually equivalent points. N, nasal; T, temporal; VM, visual midline. Modified from Ref. 15. moving objects from one visual field to the other and to integration of large-scale features across the visual field, such as optic flow during locomotion (see below; 19,20).

In the somatosensory cortex, S1, and the motor cortex, M1, callosal-projecting cells have been described to be restricted to a narrow band representing the body midline (see Ref. 12). However, more recent findings indicate that in $\mathrm{S} 1$ there are a substantial number of neurons with bilateral receptive fields in the representations of the hand and feet, which could participate in integrating information necessary for the cooperative actions of the two hands (21). These bilateral cells are probably connected to the other hemisphere via the corpus callosum. In primary and secondary auditory areas of the rat, in which different tones are represented as stripes across the respective areas, callosal cells have been described to be segregated in relation to the tonotopic representation, although there are some discrepancies in the details of this array (22-26).

Callosal projections in higher-order cortical regions have been mostly studied in the prefrontal and temporoparietal visual areas. These regions tend to be connected interhemispherically by poorly myelinated, thindiameter, slow-conducting fibers $(8,9)$. There, callosal cells tend to be distributed all over the respective cortical regions, interdigitating with ipsilateral corticocortical projections and with thalamic projections in a manner resembling the ocular-dominance array of the primary visual cortex. These higher-order cortical areas tend to have a poor visuotopic organization. Their cells have large receptive fields and respond better to stimulus properties such as color, shape or direction of movement than to strict position in the visual field (12).

\section{Visual callosal fibers: midline fusion and depth perception}

The concentration of fast-conducting, 
large-diameter callosal fibers in primary/secondary sensorimotor areas might reflect the importance of performing the fusion of the two sensory and motor spatial hemirepresentations across the hemispheres. This is especially important in two sensory systems: the auditory system, which may use binaural cells to make up a cortical sound localization system, and the visual system, in which central vision involves high-resolution processing and is related to aspects of depth perception. In fact, the callosal regions corresponding to the primary and secondary auditory and visual areas have the largest fiber diameters of the corpus callosum (9).

The role of the corpus callosum in central vision and depth perception has been debated for some time. Reports indicate that damage to the posterior corpus callosum produces inability to judge depth in patients with damage of the optic chiasm, and section of the corpus callosum may reduce the proportions of binocular cells (27-29). In addition, experimentally induced early strabismus leads to an expanded callosal receiving zone in areas 17 and 18; in these conditions callosal cells also show decreased binocularity, decreased ability to respond to fastmoving stimuli, a small receptive field size, and poor orientation selectivity (19). Midline visual deficits (30) and impairment of interhemispheric depth comparisons when using head movements to determine relative depth (31) have been reported in acallosal humans. However, other reports indicate that the corpus callosum is not critical for depth perception, and that stereoscopic vision is mostly determined by the interactions between the thalamocortical crossed and uncrossed pathways in the visual cortex of each hemisphere (32-34). It is possible that the corpus callosum is involved in more subtle mechanisms of depth perception than stereopsis (which is specifically defined as binocular disparity), such as relative motion or parallax, that is, using the differences in relative motion of near and far objects to judge depth (31). In this context, it has been suggested that visual callosal fibers participate in predicting trajectories of moving objects across the midline, and in the generation of binding mechanisms in the central visual field $(19,20)$.

Recently, we studied the fiber composition of the posterior corpus callosum in different species which differ in brain size, namely the mouse, the rabbit, the cat, the dog, the cow and the horse (10). We found that, despite a quite conservative cross-species distribution of fiber sizes, a small population of large-diameter fibers increased their caliber in species with larger brains. Furthermore, in the cat and the dog, both species with frontal vision, the largest fiber diameters were larger than expected from their brain size, as compared to species with more lateral vision (see Figure 4). This is consistent with the hypothesis that the large callosal fibers are related to aspects of central vision.

\section{Origin of the corpus callosum}

In reptiles, topographically organized sensory maps are restricted to mesencephalic levels (35), for which there is a well-developed tectal commissure (Figure 5). In these animals, the sensory projections to the telencephalon lose their topographic organization. Furthermore, interhemispheric fibers, represented by the anterior commissure and the hippocampal commissure, are quite scarce in reptiles. In contrast, in the mammalian

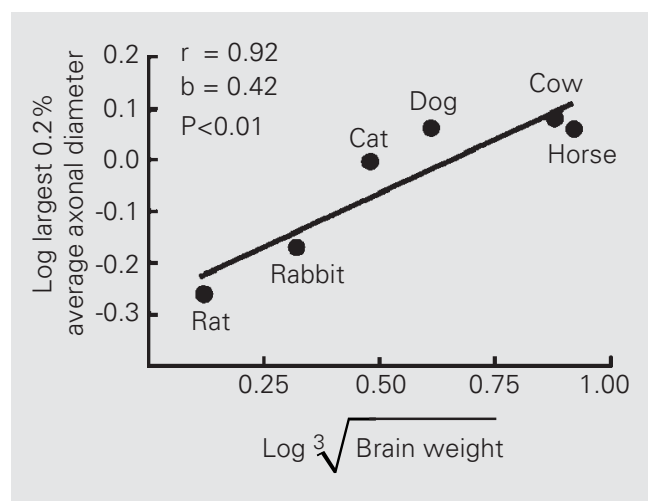

Figure 4. Relation of maximal fiber diameter in the posterior corpus callosum with brain weight of different species. Modified from Ref. 10. 
Figure 5. Topographic representation of the visual fields on both sides of the brain in reptiles and mammals. In reptiles, topographic visual projections are localized in the mesencephalic optic tectum (OT), while the telencephalic dorsal cortex (DCX) has a very poor visuotopic organization. In mammals, the superior colliculus (SC, homologous to the OT) has a visuotopic organization, but the most important topographic representation is in the primary visual cortex (VCX homologous to the DCX). The tectal commissure (TC) connects the two hemirepresentations of the visual field in the mesencephalon of reptiles and mammals, while in the mammalian visual cortex both hemirepresentations are connected via the anterior commissure (AC, marsupials and monotremes) or the corpus callosum (CC, placentals). L, left; $R$, right. telencephalon, topographically organized sensory representations take place in the multilaminar neocortex $(36,37)$. This coincides with the development of a strong commissural system in monotremes, marsupials and placentals (38).

Based on the correspondence between the origin of topographic representations in the cerebral cortex and the origin of interhemispheric fibers, and on the observation that the most important timing constraints for interhemispheric transmission may be in primary and secondary sensory areas, we suggest that the origin of the mammalian corpus callosum is related to the need to integrate the two topographic hemirepresentations of the sensory surface across the midline (Figure 5; 39). Another important early callosal function may have been bimanual coordination (21), but in our view this may have involved the corpus callosum at later stages of cortical evolution, after the topographic maps were already established and the cerebral cortex had begun to exert a significant control in motor behavior via the corticospinal tract.

In early mammals, there may have been strong selection for a system performing midline fusion in the neocortical sensory representations. There were two different
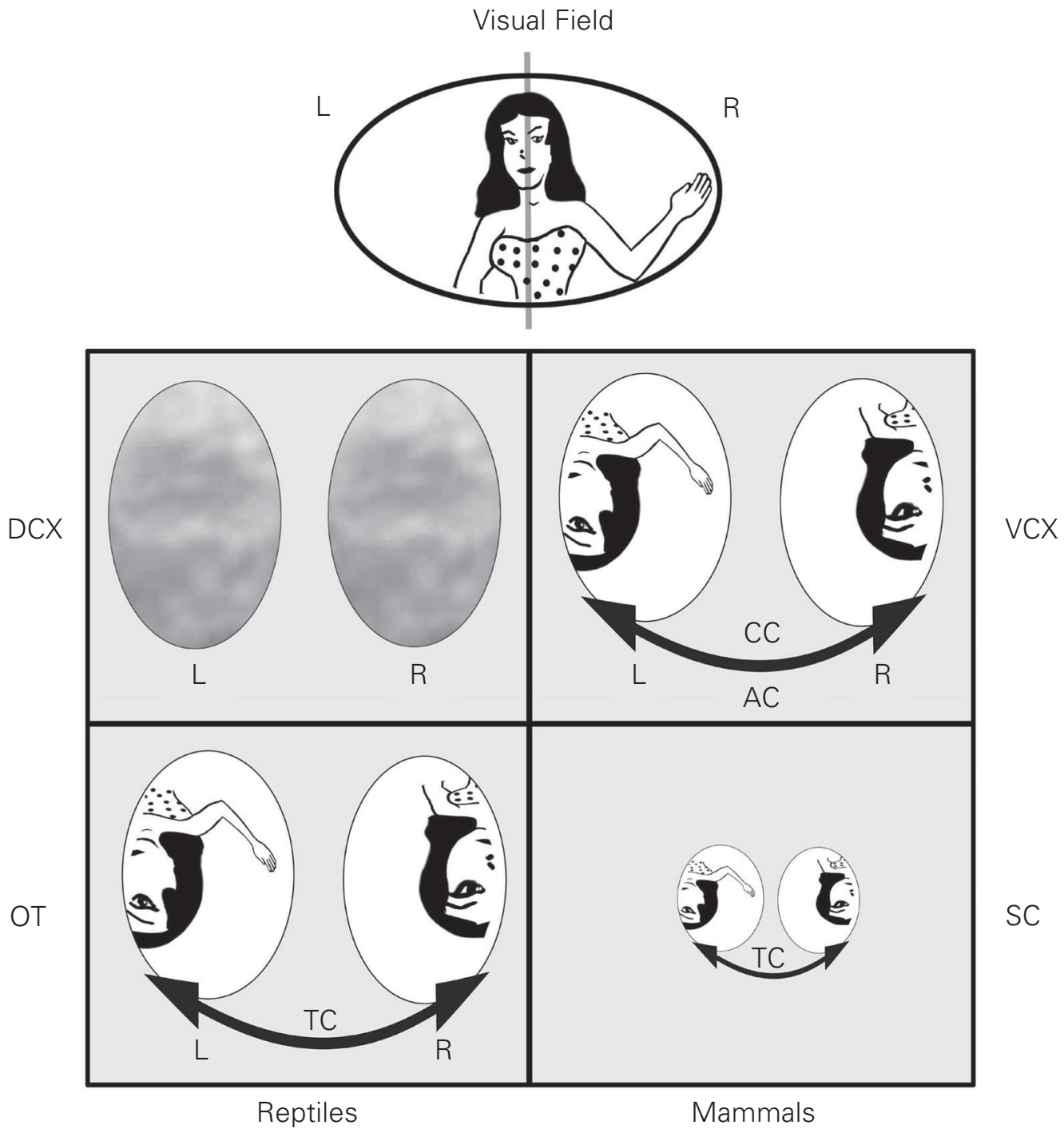
Figure 6. The corpus callosum of eutherian (placental) mammals serves as a shortcut for interhemispheric fibers. In metatherians (marsupials), fibers cross via the anterior commissure (Modified from the original drawing by $\mathrm{A}$. Frichtle, Ref. 40)

pathways that interhemispheric fibers could use to travel across the midline: the anterior commissure and the hippocampal commissure. One pathway was followed by monotremes and marsupials, in which many axons run to the other hemisphere through the anterior commissure. Consequently, this structure is notably enlarged in relation to other mammals and reptiles. This is not to say that in this group there may not be interhemispheric fibers running via the hippocampal commissure. However, the route via the anterior commissure has the disadvantage that fibers must run a long distance from the dorsally located cerebral cortex to the ventral anterior commissure, which may produce an unwanted delay in interhemispheric transmission - especially in species with large brains. In fact, in large-brained marsupials like the kangaroo, interhemispheric cortical fibers form the so-called "fasciculus aberrans", which descends from the subcortical white matter along the internal capsule and then reaches the anterior commissure, thus significantly shortening the traveling distance (Figure 6; 38). A more efficient solution to interhemispheric communication evolved in placental mammals (Figure 6) with the origin of the corpus callosum. Ontogenetically and phylogenetically, the corpus callosum originates from the hippocampal commissure and gradually separates from it, moving to a position dorsal to the hippocampal formation (38). In this position, axons travel a much shorter distance between the hemispheres than if they were to cross ventrally through the anterior commissure. In our view, interhemispheric fibers permitted the integration of both hemirepresentations of the sensory surface in the cerebral cortex,
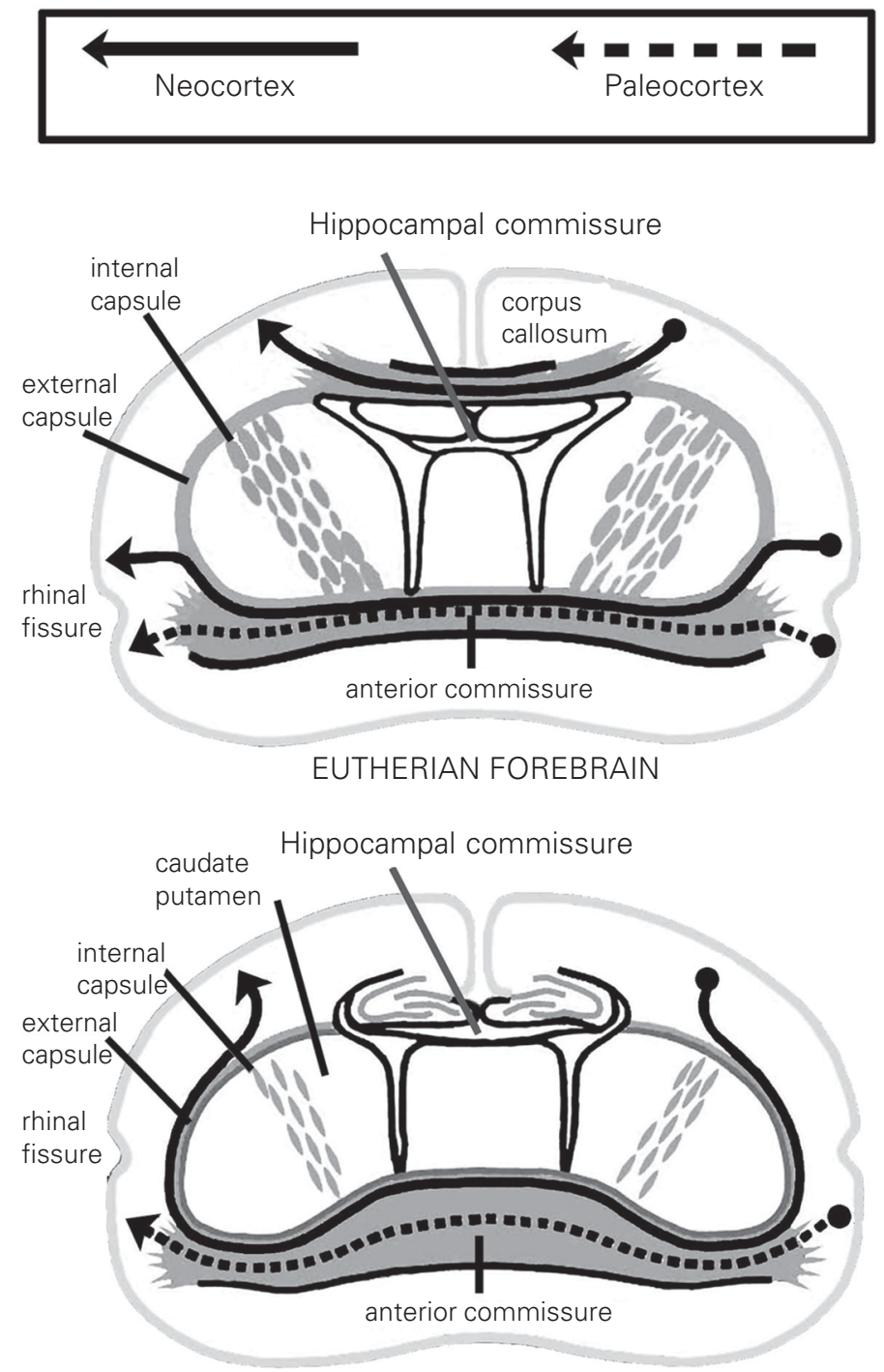

METATHERIAN FOREBRAIN (Polyprotodontia)

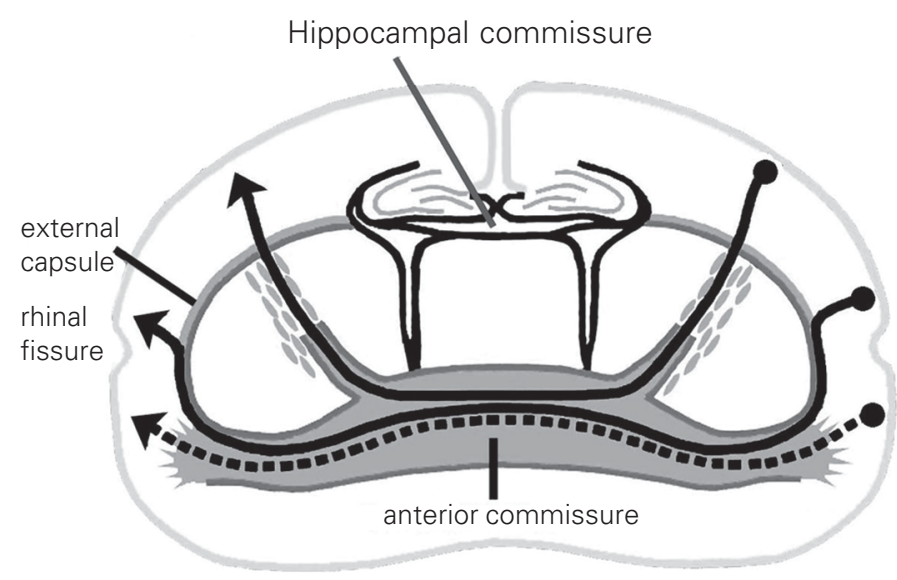

METATHERIAN FOREBRAIN (Diprotodontia) 
while the corpus callosum originated in placental mammals as a strategy to minimize interhemispheric transmission time for fibers connecting primary and secondary sensory areas.

In present day placentals, only a small contingent of callosal fibers connect primary and secondary areas. Most callosal fibers connect higher-order areas, which have a poor topographic organization. How did these fibers become so abundant if the primary function of the corpus callosum relates to midline fusion and bimanual coordination? One possibility is that they crossed the hemispheres just because they could. Despite its underlying molecular complexity, the ability to cross the telencephalic midline may be shared by many cortical axons, in fact many more than those that are finally retained in the adult. The number of callosal fibers is dramatically reduced by $75 \%$ during the perinatal period of the primate (41), and perhaps in similar proportions in other mammals. Furthermore, some callosal axons have been found to re-cross to the ipsilateral hemisphere through the anterior commissure (42). These axons retract their bicommissural extension early in the postnatal period. This indicates that there is not much specificity in terms of the kinds of fibers that will cross the midline. In other words, once a way was open for axons to cross to the other hemisphere, the axons began to use this pathway to colonize new regions. Although this may help explain why the fibers connecting higher-order areas were initially able to grow to the contralateral hemisphere, it leaves open the question of why these fibers were maintained in evolution.

\section{Factors favoring the expansion of the corpus callosum: interhemispheric synchrony}

In recent years, synchronicity in neuronal firing has been proposed as a general mechanism for neuronal processing (43-45).
Small- or large-scale groups of interconnected neurons can show synchronous oscillations in their firing activity, which enables them to work as a unified whole for a period of time. This mechanism has been proposed to account for basic aspects of perception such as binding (that is, the process by which the information of color, movement and form become integrated into unified percepts) and for more general phenomena like consciousness. In the cat, callosal-dependent interhemispheric synchrony has been observed during visual stimulation (46), and in humans, synchronized activity in the gamma band $(40 \mathrm{~Hz})$ has been found to spread across the hemispheres during a visual recognition task (47), which may be mediated by the corpus callosum. Furthermore, analyses of the morphology and diameter of callosal axonal branches suggest that the architecture of callosal axons is suitable to promote the synchronous activation of multiple targets in the opposite hemisphere (16). It would not be surprising that processes such as depth perception or binding in the central visual field depend on the generation of synchronous ensembles of neuronal activity in the two hemispheres, for which callosal fibers may be fundamental (20).

Although originally the functions of callosal fibers may have been related to aspects of midline fusion, in later stages they may have participated in spreading corticocortical synchronous activation to other cortical areas. In most cases, callosal axons have widespread arbors that terminate in many regions beside the topographically equivalent one (16). Thus, heterotopic callosal projections (connecting nonequivalent areas in the two hemispheres) may be very important to propagate activity to other cortical areas. This may have contributed to the generation of largescale, bi-hemispheric neuronal ensembles of corticocortical interactions which participate in diverse aspects of cortical processing (44). In other words, the cortical spread of largescale cortical assemblies may have been fa- 
cilitated through a mechanism of interhemispheric reciprocal interactions. In a way, this may be similar to the callosal propagation of an epileptic focus; interhemispheric reciprocal excitation works as a facilitatory mechanism for the seizure, which spreads to the ipsilateral territory in both hemispheres. In fact, in patients callosotomy has proved to be an effective treatment to prevent the expansion of epileptic activity (4). It is therefore possible that interhemispheric connections may be advantageous for the spread of normal synchronous activity in the cerebral cortex.

\section{Relation to brain lateralization}

Some years ago, a controversial hypothesis suggested that in large-brained species, interhemispheric transmission time would be increased due to the longer distances needed to reach the contralateral hemisphere; this would promote interhemispheric isolation and consequently the development of brain lateralization (48). We and others have determined that callosal fiber composition tends to be conservative across species $(10,49)$, indicating that interhemispheric transmission time can be significantly impaired in large-brained species (50). There is only a small contingent of fibers of very wide diameter whose caliber increases with increasing brain weight, but their increase in maximal diameter may not be sufficient to fully compensate for the increasingly long interhemispheric distances (10). Furthermore, across species the proportion of callosal fibers in relation to brain size or to the estimated numbers of cortical cells tends to decrease with increasing brain weight $(10,51$, 52 ), thereby reducing the degree of interhemispheric connectivity. Overall, this evidence suggests that reduced callosal transmission is related to increased brain size, which is consistent with the hypothesis of Ringo et al. (48).

This discussion is also relevant to the issue of interhemispheric synchrony. One important requisite for the development of synchronous ensembles, especially largescale ones, is a high transmission velocity across distant regions in the brain (43). In small-brained mammals this may not be a significant problem because the axonal distances are short, but in larger-brained animals like humans, the interhemispheric distances may become so large as to somewhat impair callosal transmission. It is possible that in large-brained animals, synchronous activity mediated by the corpus callosum can be achieved only by specialized "channels" provided by a few fast-conducting fibers (53). In this context, it is of interest that in all callosal regions the proportions of large-diameter fibers in the corpus callosum tend to increase with age $(54,55)$. This may imply that these fibers participate in the generation of interhemispheric ensembles which become stabilized during late ontogeny. In any case, the size-related constraints for interhemispheric synchrony may have well facilitated the development of intrahemispheric ensembles, which may have promoted the development of brain lateralization. In this sense, although the corpus callosum may have contributed to the interhemispheric spread of synchronous ensembles in small mammals, it is possible that a point was reached in which, beyond a given brain size, problems appeared for the transmission of some types of information between the hemispheres. Even considering that interhemispheric synchronous activity is possible in humans and other animals, the question still remains as to whether in our species there are certain subtle limiting factors in the generation of interhemispheric synchrony. These may have facilitated the development of strong, intrahemispheric neuronal loops, thus promoting hemispheric independence and the establishment of brain lateralization.

Finally, in humans, a male-specific inverse relation between brain lateralization and callosal connectivity has been docu- 
mented in several studies $(9,56,57)$. However, it is not clear if in humans increased brain lateralization is caused by a decrease in interhemispheric communication or vice versa. One possibility is that the two factors reinforce each other in development, that is, an initially increased anatomical brain asymmetry may produce a larger than normal retraction of callosal axons, which in turn may promote interhemispheric isolation, thus strengthening the incipient brain laterality $(39,58)$.

\section{Final comment}

In this article we have intended to provide a scenario for the origins and evolution of the corpus callosum in placental mammals. Interhemispheric fibers possibly originated in mammals as a consequence of the development of the isocortex and of topographic maps of the sensory and motor surfaces in it. In monotremes and marsupials, fibers travel to the other hemisphere via the anterior commissure and the hippocampal commissure. In placental mammals, the corpus callosum originates above the hippocampal commissure as the main tract connecting the left and right cortices, and may provide a shortcut for interhemispheric axons to the contralateral hemisphere. Commis- sural fibers may have first participated in binding mechanisms related to midline fusion and bimanual coordination, an ability observed in many so-called primitive mammals. The lack of specificity by which cortical axons may cross through the corpus callosum allowed many kinds of fibers connecting diverse areas of the cerebral cortex, especially through heterotopic connections, which contributed to the spread of neuronal ensembles in different aspects of cognitive processing. In species with large brain size like humans, constraints may have appeared limiting conduction speed and the amount of interhemispheric fibers, thus favoring intrahemispheric processing and the development of brain lateralization. Although some of these ideas may not be testable, there are proposals that may provide ideas for future research. For example, it will be interesting to compare the tectal commissure of reptiles and the visual callosal fibers of mammals, as well as the role of the large-diameter visual fibers in central vision. Furthermore, the role of the corpus callosum in the generation of large-scale neuronal ensembles remains to be demonstrated, and further studies are needed to ascertain the role of interhemispheric communication in the development of brain lateralization.

\section{References}

1. Huxley TH (1863). Man's Place in Nature. McMillan, London, UK.

2. Flower WH (1865). On the commissures of the cerebral hemispheres of the Marsupialia and Monotremata as compared with those of placental mammals. Philosophical Transactions of the Royal Society of London, 23: 633-651.

3. Smith E (1910). Some problems relating to the evolution of the brain. Lancet, 1: 1-6.

4. Gazzaniga MS (2000). Cerebral specialization and interhemispheric communication. Does the corpus callosum enable the human condition? Brain, 123: 1293-1326.

5. Schalomon PM \& Wahlsten D (2002). Wheel running behavior is impaired by both surgical section and genetic absence of the mouse corpus callosum. Brain Research Bulletin, 57: 27-33.

6. Rockland KS \& Pandya DN (1986). Topography of occipital lobe commissural connections in the rhesus monkey. Brain Research,
365: $174-178$

7. Kim JH, Ellman A \& Juraska JM (1996). A re-examination of sex differences in axon density and number in the splenium of the rat corpus callosum. Brain Research, 740: 47-56.

8. LaMantia AS \& Rakic P (1990). Cytological and quantitative characteristics of four cerebral commissures in the rhesus monkey. Journal of Comparative Neurology, 291: 520-537.

9. Aboitiz F, Scheibel AB, Fisher RS \& Zaidel E (1992). Fiber composition of the human corpus callosum. Brain Research, 598: 143-153.

10. Olivares R, Montiel J \& Aboitiz F (2001). Species differences and similarities in the fine structure of the mammalian corpus callosum. Brain, Behavior and Evolution, 57: 98-105.

11. Lomber SG, Payne BR \& Rosenquist AC (1994). The spatial relationship between the cerebral cortex and fiber trajectory through the corpus callosum of the cat. Behavioural Brain Research, 64: 25-35. 
12. Innocenti GM (1986). General organization of callosal connections in the cerebral cortex. In: Jones EG \& Peters A (Editors), Cerebral Cortex. Vol. 5. Plenum Press, New York, NY, USA, 291-354.

13. Innocenti GM, Aggoun-Zouaoui D \& Lehmann P (1995). Cellular aspects of callosal connections and their development. Neuropsychologia, 33: 961-988.

14. Olavarría JF (1996). Non-mirror symmetric patterns of callosal linkages in areas 17 and 18 in cat visual cortex. Journal of Comparative Neurology, 366: 643-655.

15. Olavarría JF (2001). Callosal connections correlate preferentially with ipsilateral cortical domains in cat areas 17 and 18, and with contralateral domains in the $17 / 18$ transition zone. Journal of Comparative Neurology, 433: 441-457.

16. Houzel JC \& Milleret C (1999). Visual inter-hemispheric processing: constraints and potentialities set by axonal morphology. Journal of Physiology, 93: 271-284.

17. Berlucchi G (1972). Anatomical and physiological aspects of visual function of the corpus callosum. Brain Research, 214: 239-259.

18. Olavarría JF \& Van Sluyters RC (1983). Widespread callosal connections in infragranular visual cortex of the rat. Brain Research, 279: 233-237.

19. Milleret C \& Houzel JC (2001). Visual interhemispheric transfer to areas 17 and 18 in cats with convergent strabismus. European Journal of Neuroscience, 13: 137-152.

20. Houzel JC, Carvalho ML \& Lent R (2002). Interhemispheric connections between primary visual areas: beyond the midline rule. Brazilian Journal of Medical and Biological Research, 35: 1441-1453.

21. Iwamura $Y$ (2000). Bilateral receptive field neurons and callosal connections in the somatosensory cortex. Philosophical Transactions of the Royal Society of London, Series B. Biological Sciences, 355: 267-273.

22. Imig TJ \& Bruge JF (1978). Sources and terminations of callosal axons related to binaural and frequency maps in primary auditory cortex of the cat. Journal of Comparative Neurology, 182: 637-660.

23. Pallas SL, Littman T \& Moore DR (1999). Cross-modal reorganization of callosal connectivity in auditory cortex without altering thalamocortical projections. Proceedings of the National Academy of Sciences, USA, 96: 8751-8756.

24. Pallas SL (2001). Intrinsic and extrinsic factors that shape neocortical specification. Trends in Neurosciences, 24: 417-425.

25. Scheich H, Heil P \& Langner G (1993). Functional organization of auditory cortex in the Mongolian gerbil (Meriones unguiculatus). II. Tonotopic 2-deoxyglucose. European Journal of Neuroscience, 5: 898-914

26. Thomas H, Tillein J, Heil P \& Scheich H (1993). Functional organization of auditory cortex in the Mongolian gerbil (Meriones unguiculatus). I. Electrophysiological mapping of frequency representation and distinction of fields. European Journal of Neuroscience, 5: 882897.

27. Elberger AJ \& Smith EL (1985). The critical period for corpus callosum section to affect cortical binocularity. Experimental Brain Research, 57: 213-223.

28. Gardner JC \& Cynader MS (1987). Mechanisms for binocular depth sensitivity along the vertical meridian of the visual field. Brain Research, 413: 60-74.

29. Hubel DH (1988). Eye, Brain and Vision. Scientific American Library, New York, NY, USA, 156-157.

30. Jeeves MA (1991). Stereo perception in callosal agenesis and partial callosotomy. Neuropsychologia, 29: 19-34.

31. Rivest J, Cavanagh P \& Lassonde M (1994). Interhemispheric depth judgment. Neuropsychologia, 32: 69-76.
32. Lepore F, Ptito M \& Lassonde M (1986). Stereoperception in cats following section of the corpus callosum and/or the optic chiasma. Experimental Brain Research, 61: 258-264.

33. Ptito M, Lepore F \& Guillemot JP (1991). Stereopsis in the cat: behavioral demonstration and underlying mechanisms. Neuropsychologia, 29: 443-464.

34. Cowey A \& Wilkinson F (1991). The role of the corpus callosum and extra striate visual areas in stereoacuity in macaque monkeys. Neuropsychologia, 29: 465-479.

35. Ten Donkelaar HJ (1998). Reptiles. In: Nieuwenhuys R, Ten Donkelaar HJ \& Nicholson C (Editors), The Central Nervous System of Vertebrates. Springer-Verlag, Berlin, Germany.

36. Aboitiz F (1999). Evolution of isocortical organization. A tentative scenario including roles of reelin, p35/cdk5 and the subplate zone. Cerebral Cortex, 9: 655-661.

37. Aboitiz F, Morales D \& Montiel J (2001). The inverted neurogenetic gradient of the mammalian isocortex: development and evolution. Brain Research Reviews, 38: 129-139.

38. Abbie AA (1939). The origin of the corpus callosum and the fate of the structures related to it. Journal of Comparative Neurology, 70: 944.

39. Aboitiz F \& Ide A (1998). Anatomical asymmetries in languagerelated cortex and their relation to callosal function. In: Stemmer E \& Whitaker $\mathrm{H}$ (Editors), Handbook of Neurolinguistics. Academic Press, New York, NY, USA, 393-404.

40. Shang F, Ashwell KWS, Marotte LR \& Waite PME (1997). Development of commissural neurons in the Wallaby (Macropus eugenii). Journal of Comparative Neurology, 387: 507-523.

41. LaMantia AS \& Rakic P (1990). Axon overproduction and elimination in the corpus callosum of the developing rhesus monkey. Journal of Neuroscience, 10: 2156-2175.

42. Hedin-Pereira C, Uziel D \& Lent R (1992). Bicommissural neurones in the cerebral cortex of developing hamsters. NeuroReport, 3: 873876.

43. Singer W (1999). Neuronal synchrony: a versatile code for the definition of relations? Neuron, 24: 49-65, 111-125.

44. Varela FJ, Lachaux J-P, Rodríguez E \& Martinerie J (2001). The brainweb: phase synchronization and large-scale integration. Nature Reviews. Neuroscience, 2: 229-239.

45. Engel AK, Fries $P$ \& Singer W (2001). Dynamic predictions: oscillations and synchrony in top-down processing. Nature Reviews. Neuroscience, 2: 704-716.

46. Engel AK, König P, Kreiter AK \& Singer W (1991). Interhemispheric synchronization of oscillatory neural responses in cat visual cortex. Science, 252: 1177-1179.

47. Rodríguez E, George N, Lachaux J-P, Martinerie J, Renault B \& Varela FJ (1999). Perception's shadow: long-distance gamma band synchronization of human brain activity. Nature, 397: 430-433.

48. Ringo JL, Doty RW, Demeter S \& Simard PY (1994). Time is of the essence: a conjecture that hemispheric specialization arises from interhemispheric conduction delay. Cerebral Cortex, 4: 331-343.

49. Schüz A \& Preissl H (1996). Basic connectivity of the cerebral cortex and some considerations on the corpus callosum. Neuroscience and Biobehavioral Reviews, 20: 567-570.

50. Aboitiz F, Montiel J \& López J (2002). A hypothesis on the early evolution of the develoment of the isocortex. Brain Research Bulletin, 57: 481-483.

51. Rilling JK \& Insel TR (1999). Differential expansion of neural projection systems in primate brain evolution. NeuroReport, 10: 14531459.

52. Olivares R, Michalland S \& Aboitiz F (2000). Cross-species and 
intraspecies morphometric analysis of the corpus callosum. Brain, Behavior and Evolution, 55: 37-43.

53. Aboitiz F, López J \& Montiel J (2002). Long distance communication in the human brain: timing constraints for interhemispheric synchrony and the origin of brain lateralization. Biological Research (in press).

54. Aboitiz F, Rodríguez E, Olivares R \& Zaidel E (1996). Age-related changes in fiber composition of the human corpus callosum. NeuroReport, 7: 1761-1764

55. Godlewski A (1991). Morphometry of myelin fibers in corpus callosum and optic nerve of aging rats. Journal für Hirnforschung, 83:
568-581.

56. Dorion AA, Chantome M, Hasboun D, Zouaoui A, Marsault C, Capron C \& Duyme M (2000). Hemispheric asymmetry and corpus callosum morphometry: a magnetic resonance imaging study. Neuroscience Research, 36: 9-13.

57. Witelson SF \& Goldsmith CH (1991). The relationship of hand preference to anatomy of the corpus callosum in men. Brain Research, 545: 175-182

58. Aboitiz F, Scheibel AB \& Zaidel E (1992). Morphometry of the Sylvian fissure and the corpus callosum, with emphasis on sex differences. Brain, 115: 1521-1541. 